\title{
Evaluation of the lateral sentinel node by indocyanine green for rectal cancer based on micrometastasis determined by reverse transcriptase-polymerase chain reaction
}

\author{
SHINGO NOURA ${ }^{1}$, MASAYUKI OHUE ${ }^{1}$, YOSUKE SEKI ${ }^{1}$, TAKASHI YAMAMOTO ${ }^{2}$, \\ ATSUSHI IDOTA ${ }^{2}$, JUNKO FUJII ${ }^{2}$, TOMOYUKI YAMASAKI ${ }^{2}$, HIROMU NAKAJIMA ${ }^{2}$, \\ KOHEI MURATA ${ }^{3}$, MASAO KAMEYAMA ${ }^{4}$, TERUMASA YAMADA ${ }^{1}$, ISAO MIYASHIRO ${ }^{1}$, \\ HIROAKI OHIGASHI $^{1}$, MASAHIKO YANO ${ }^{1}$, OSAMU ISHIKAWA ${ }^{1}$ and SHINGI IMAOKA ${ }^{1}$ \\ Departments of ${ }^{1}$ Surgery and ${ }^{2}$ Clinical Laboratory, Osaka Medical Center for Cancer and \\ Cardiovascular Diseases, Osaka; ${ }^{3}$ Department of Surgery, Suita Municipal Hospital, \\ Suita; ${ }^{4}$ Department of Surgery, Bell Land General Hospital, Sakai, Japan
}

Received April 17,2008; Accepted May 27, 2008

DOI: 10.3892/or_00000069

\begin{abstract}
The significance of dissecting the lateral pelvic lymph node (LN) for lower rectal cancer remains controversial. We detected the lateral sentinel node ( $\mathrm{SN}$ ) by indocyanine green (ICG) and micrometastases using carcinoembryonic antigen (CEA)-specific reverse transcriptase-polymerase chain reaction (RT-PCR). Twenty-five patients who underwent curative surgery with a dissection of the lateral pelvic LNs between 2003 and 2005 were examined. We investigated the existence of lateral SNs and any associations between pathological metastases and micrometastases by RT-PCR. Lateral SNs were detected in 7 (28\%) of the 25 patients. The number of lateral SNs was $13 \mathrm{LNs}$, or 1.9 nodes per case. Of the 25 cases, 7 had lateral LN metastases based on pathological examinations in dissected lateral LNs. Three cases had massive lateral LN swelling by pre-operative pelvic $\mathrm{CT}$ and the SNs were not detected in them. The SNs were detected in two cases and were negative based on pathological examinations and positive according to a genetic diagnosis. SNs were detected in one case, which was positive based on pathological examinations and a genetic diagnosis. SN was not detected in one case. There were five SNs in which CEA was positive by RT-PCR, though only one of them was positive based on pathological examinations. No SNs were observed that were negative based on a genetic diagnosis, but were positive according to the pathological diagnosis. We
\end{abstract}

Correspondence to: Dr Shingo Noura, Department of Surgery, Osaka Medical Center for Cancer and Cardiovascular Diseases, 1-3-3 Nakamichi, Higashinari-ku, Osaka 537-8511, Japan

E-mail: noura-si@mc.pref.osaka.jp

Key words: rectal cancer, lateral sentinel node, indocyanine green, micrometastasis, reverse transcriptase-polymerase chain reaction detected the lateral SNs using ICG. The sensitivity of identifying lateral LN metastasis was improved by the use of a genetic diagnosis. However, the detection rate was still low therefore we need to develop a new method for detecting SNs.

\section{Introduction}

Metastasis to the regional lymph node (LN) is an important prognostic factor, which is used for clinical decision-making regarding the selection of the most appropriate cancer treatment. The development of locoregional recurrences and distant metastases accounts for most of the deaths. Adequate tumor staging, including an evaluation of the presence of regional LN metastases at the time of diagnosis, is essential, since it constitutes the most important prognostic factor (1-5). Local recurrence develops in about half of all patients with advanced rectal cancer, especially in the lower rectum, who underwent a potentially curative resection (6). Although many trials consisting of extended surgery, adjuvant chemotherapy and radiotherapy were carried out to prevent local recurrence after curative surgery, no significantly favorable results have yet been reported. Since the early 1980s, the results of a lateral pelvic LN dissection (LPLD) for rectal cancer have improved steadily, although LPLD performed in Japan at that time often caused urinary and sexual dysfunction $(7,8)$. Since the beginning of the mid 1980s, colorectal surgeons in Japan have sought to improve their surgical techniques to either alleviate or prevent functional disturbances and to individualize the operative procedures according to the local extent of the cancer without increasing the risk of local recurrence. These efforts have led to the development of various procedures developed with the goal of pelvic autonomic nerve preservation (9).

The sentinel node (SN) concept was first advocated by Morton et al (10) in patients with melanoma. Sentinel node navigation surgery (SNNS) for breast cancer and malignant melanoma can accurately assess LN dissection areas $(11,12)$. The SN concept was recently applied to cancers of 
the gastrointestinal tract (13-16). If SNNS can be applied to lower rectal cancer, unnecessary LPLD can be omitted and personalized lymphadenectomy may be possible.

Some authors reported the clinical significance of LN micrometastasis detected by either immunohistochemistry (IHC) or reverse transcriptase-polymerase chain reaction (RT-PCR) (17-20). The aim of this study was to investigate whether the SN concept by indocyanine green (ICG) is a useful indicator of lateral region lymph node involvement in lower rectal cancer patients. In addition, we investigated the significance of the lateral SNs for lower rectal cancer based on micrometastasis as determined by RT-PCR.

\section{Patients and methods}

Patients. In our hospital, Stage II or III [clinically staged according to the tumor node metastasis (TNM) classification of UICC (Union International Contre le Cancer) (21)] middle or lower rectal cancer, located at or below the peritoneal reflection, is an indication of LPLD. Twenty-five patients with lower rectal cancer, who were preoperatively diagnosed to have Stage II or III of the disease, were investigated. The patients were clinically diagnosed before surgery based on the findings of a colonoscopy, Ba-enema, CT and MRI. All underwent curative surgery with LPLD at our department of surgery between January 2003 and April 2005. These patients $(n=25)$ included $12(48 \%)$ males and $13(52 \%)$ females, with a mean age of $58.8 \pm 10.5$ years $( \pm \mathrm{SD}$, range, 41 to 77 years). The tumor lower levels were located at or below the peritoneal reflection. None of the patients had undergone either preoperative chemotherapy or irradiation (Table I). This study was approved by the Human Ethics Review Committee of the Osaka Medical Center for Cancer and Cardiovascular Diseases and a signed informed consent form was obtained from each patient.

Detection of the lateral SNs. Before laparotomy, a fine needle (26-gauge) was inserted into the submucosal layer via the anus at three sites anal sides of the tumor circumferentially, and dye, indocyanine green (ICG) (Diagnogreen; Dai-Ichi Pharm Co Ltd, Tokyo, Japan) was then gently injected. The total amount of injected dye was $5 \mathrm{ml}(25 \mathrm{mg})$ for each patient. After the laparotomy, the lateral vesical and obturator spaces were opened between the lateral aspect of the internal iliac vessels and the pelvic wall. In addition, we observed the lateral pelvic adipose tissue around the internal iliac arteries and the obturator spaces with macroscopic observation. Sixty minutes after the ICG injection, we were able to identify the SNs around a lateral lesion and then performed a rectal resection and lateral pelvic LN dissection with autonomic nerve preservation. Even though we could not detect the lateral SN, we performed the same operation.

Tissue preparations. Each SN was immediately cut into halves individually to prevent RNA cross-contamination between specimens. One half of the node was fixed with $10 \%$ buffered formalin and was then embedded in paraffin for $\mathrm{H} \& \mathrm{E}$ staining. The other half was stored in RNALater solution (Ambion, Austin, TX) at $-20^{\circ} \mathrm{C}$ until RNA extraction.
RNA extraction from $S N$ specimens and RT-PCR. Tissue specimens were minced with RNase-free disposable pellet pestles (Kimble Kontes, Vineland, NJ) in TRIzol Reagent (Invitrogen, Carlsbad, CA). RNA extraction was carried out according to the protocol recommended by the manufacturer. Purified RNA was quantified and assessed for purity by UV spectrophotometry. Complementary DNA (cDNA) was generated with a transcriptor first-strand cDNA synthesis kit (Roche Diagnostics, Mannheim, Germany) according to the protocol provided by the manufacturer. The RT reaction was performed in a total of $20 \mu 1$ at $50^{\circ} \mathrm{C}$ for $60 \mathrm{~min}$, followed by heating at $85^{\circ} \mathrm{C}$ for $5 \mathrm{~min}$.

All PCR analyses were performed using LightCycler (Roche Diagnostics) and a real-time monitoring thermal cycler, for the rapid and quantitative detection of cancer cells. The integrity of the RNA samples was verified by the amplification of B-2-microglobulin (B2M) mRNA.

The primer sequences used for CEA detection were 5'-ccc gcagtattcttggcgtatc-3' and 5'-tgcaaatgctttaaggaagaagc-3'. The primer sequences used for B2M detection were 5'-gctatgtgtct gggtttc-3' and 5'-tacatgtctcgatcccac-3'.

For each PCR amplification, a reaction mixture was prepared containing $2 \mu \mathrm{l}$ of a cDNA template, 10X PCR buffer, $3 \mathrm{mM} \mathrm{MgCl} 2,0.25 \mu \mathrm{M}$ of primer pairs and FastStart DNA Master SYBR-Green I (Roche Diagnostics). The PCR cycling conditions were set as follows: an initial denaturing step at $95^{\circ} \mathrm{C}$ for $10 \mathrm{~min}$ and 40 cycles at $95^{\circ} \mathrm{C}$ for $15 \mathrm{sec}, 62^{\circ} \mathrm{C}$ for $10 \mathrm{sec}$ and $72^{\circ} \mathrm{C}$ for $5 \mathrm{sec}$. The quantification data were analyzed using the Light Cycler analysis software program (Roche Diagnostics $\mathrm{GmbH}$ ) as recommended by the manufacturer. The standard curves for the quantification of CEA or B2M mRNAs were drawn using 10-fold dilutions of cDNA from the human pancreatic cancer cell line CaPan1.

Cell lines and cell culture conditions. The human pancreatic cancer cell line, CaPan1, was obtained from the Health Science Research Resources Bank (Tokyo, Japan). It was grown in Dulbecco's modified Eagle's medium plus $10 \%$ fetal bovine serum, $100 \mathrm{U} / \mathrm{ml}$ penicillin and $100 \mu \mathrm{g} / \mathrm{ml}$ streptomycin and $0.25 \mu \mathrm{g} / \mathrm{ml}$ amphotericin $\mathrm{B}$, in $5 \% \mathrm{CO}_{2}$ at $37^{\circ} \mathrm{C}$.

Statistical analysis. Statistical analysis was performed with the software package StatView version 5.0 (Abacus Concepts, Inc. Berkeley, CA). The associations between the discrete variables were assessed using the Fisher exact test, the Chi-square test or Student's t-test as appropriate. P-values of $<0.05$ denoted the presence of a statistically significant difference.

\section{Results}

RT-PCR sensitivity in detecting cancer cells. Serial dilutions of CaPan 1 cancer cells $\left[1 \times 10^{0}(=1)-1 \times 10^{4}\right]$ were mixed with lymphocytes $\left(1 \times 10^{7}\right)$ obtained from healthy volunteers (that did not express CEA mRNA). Total RNA was extracted from each mixture and subjected to a quantitative RT-PCR assay. One hundred cancer cells mixed with $10^{7}$ normal lymphocytes were detected by RT-PCR with a CEA marker (Fig. 1). 

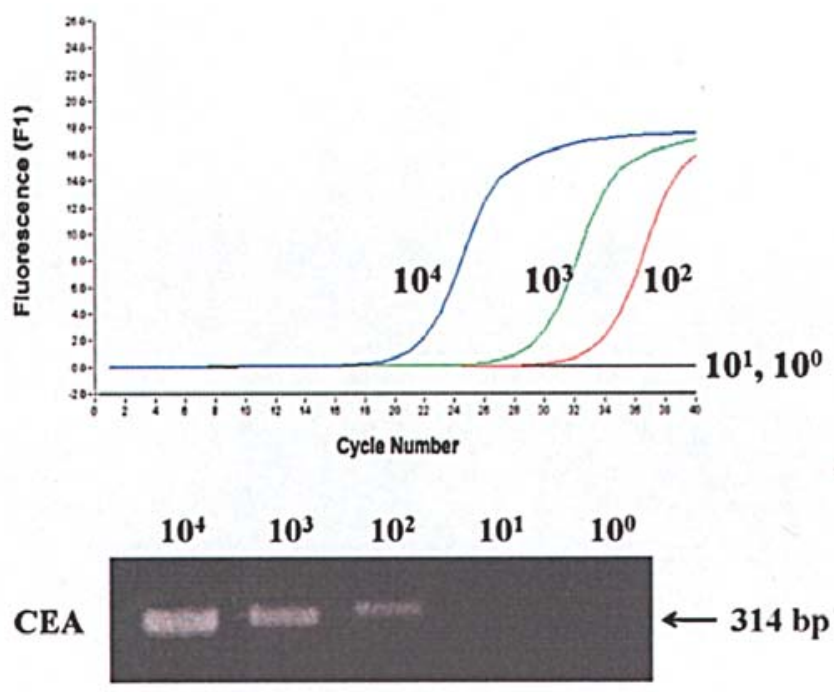

Figure 1. Real-time RT-PCR for CEA (carcinoembryonic antigen) mRNA. We used a LightCycler to detect various ratios of CaPan1 cells mixed with lymphocytes. Upper panel: fluorescence vs. PCR cycles. CaPan1 cells were sequentially diluted 10 -fold and mixed with lymphocytes to give $10^{4}$ to $10^{0} \mathrm{CaPan} 1$ cells per $10^{7}$ lymphocytes. Lower panel: ethidium bromidestained agarose gels following electrophoresis of CEA RT-PCR products. The RT-PCR products for CEA were resolved as 314 base pair fragments. bp, base pairs.

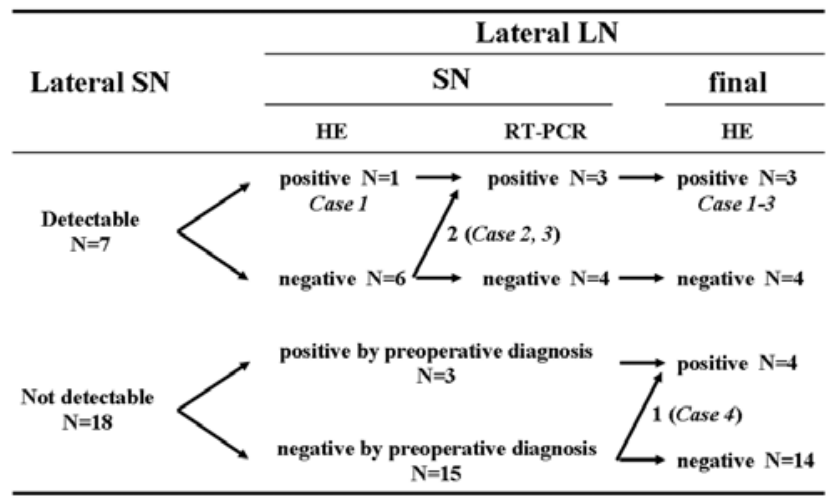

Figure 2. Distribution of lateral lymph node metastasis and micrometastasis in the sentinel and other nodes.

Detection of lateral SNs. With macroscopic observations, we detected the green-stained SNs in the lateral pelvic adipose tissue around the internal iliac arteries and the obturator spaces. We identified the lateral SNs in seven patients (28\%), a mean number of 1.9 (range, 1-4). The detection rates were $20 \%(1 / 5)$ in T2 and $30 \%(6 / 20)$ in T3 rectal cancer. No significant differences were observed in age, gender, tumor size, histological grade, LN stage, TNM stage, lymphatic invasion or venous invasion between the two groups (Table I).

Comparison of the histological and genetic diagnoses. In lateral SNs detectable cases $(\mathrm{N}=7)$, only one case (Case 1) demonstrated metastasis by HE in the lateral SNs. Another six cases demonstrated no metastasis by HE in the lateral SNs. Two cases (Case 2,3) had no metastasis by HE in the lateral SNs. However, these cases had micrometastasis by RT-PCR in the lateral SNs. Three cases (Cases 1-3) had metastasis by (a) Case 1

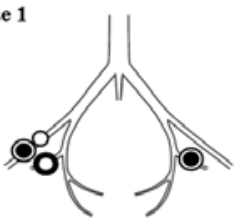

(c) Case 3

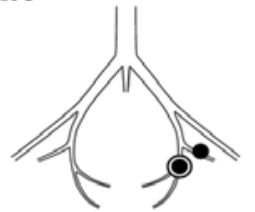

O SN, negative by both RT-PCR and HE

O SN, positive by both RT-PCR and HE (b) Case 2

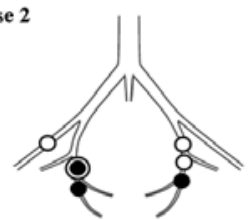

(d) Case 4

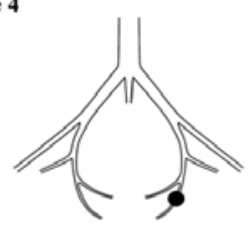

- Non-SN, positive by $\mathrm{HE}$

SN, positive by RT-PCR only
Figure 3. Anatomical mapping of the lateral LN detected by RT-PCR and histological examinations in four representative cases. (a) Case 1 had four lateral SNs. One SN was positive according to RT-PCR and HE. (b) Case 2 had four lateral SNs. One SN was positive based on RT-PCR only. (c) Case 3 had one lateral SN. One SN was positive according to RT-PCR only. One dissected lateral non-SN was found to have metastasis by HE. (d) Case 4 had no SNs. However, one lateral LN had metastasis based on HE.

HE in dissected lateral LNs. The status of dissected lateral LN metastasis is completely consistent with the diagnosis of the lateral SNs according to RT-PCR. Case 1 has four lateral SNs. One SN is positive by RT-PCR and HE. However, the other SN was negative according to RT-PCR and HE. Further two SNs were positive by RT-PCR only. In addition, the dissected lateral non-SNs demonstrated no metastasis by HE. Case 2 had four lateral SNs. One SN was positive according to RT-PCR only. However, the other three SNs were negative by RT-PCR and HE. In dissected lateral LN, two non-SNs had metastasis by HE. Case 3 had one lateral SN. One $\mathrm{SN}$ is positive by RT-PCR only. In the dissected lateral LN, one non-SN had metastasis according to HE.

In cases where the lateral $\mathrm{SNs}$ were not detectable $(\mathrm{N}=18)$, three out of 18 patients had massive lateral pelvic $\mathrm{LN}$ swelling based on pre-operative pelvic $\mathrm{CT}$ and the $\mathrm{SN}$ was not detected macroscopically. Another patient had no lateral LN swelling by pre-operative diagnosis. In case 4 , only one lateral LN along the internal iliac artery had metastasis by HE. This LN could not be detected by either pre-operative CT or MRI and could not be found by intra-operative observations (Figs. 2 and 3).

The number of lateral SNs was 13 LNs. There were five SNs in which CEA was positive by RT-PCR, though there was only one that was positive based on pathological examinations. In contrast, no SNs that were negative based on a genetic diagnosis were found to be positive according to a pathological diagnosis.

\section{Discussion}

One of the most common sites of recurrence after a curative resection of rectal cancer is the pelvis (22) and local control is a major goal of surgical treatment. Appropriate dissection in order to eliminate residual cancer foci, such as any involved LNs, is therefore necessary to achieve local control. However, the target region for local recurrence remains controversial. Some Japanese surgeons place great emphasis 
Table I. Clinicopathological findings of 25 patients with rectal cancer.

\begin{tabular}{|c|c|c|c|}
\hline & Lateral SN (+) $(\mathrm{N}=7)$ & Lateral SN (-) $(\mathrm{N}=18)$ & P-value \\
\hline Age (yrs) & $55.9 \pm 10.9$ & $59.9 \pm 10.5$ & 0.3950 \\
\hline \multicolumn{4}{|l|}{ Gender } \\
\hline Male & $3(43 \%)$ & $9(50 \%)$ & \multirow[t]{2}{*}{0.9999} \\
\hline Female & $4(57 \%)$ & $9(50 \%)$ & \\
\hline Tumor size $(\mathrm{cm})$ & $5.8 \pm 1.6$ & $5.8 \pm 1.9$ & 0.9534 \\
\hline \multicolumn{4}{|l|}{ Histological grade } \\
\hline Well & $1(14 \%)$ & $6(33 \%)$ & \multirow[t]{3}{*}{0.3418} \\
\hline Moderate & $6(86 \%)$ & $10(56 \%)$ & \\
\hline Mucinous & $0(0 \%)$ & $2(11 \%)$ & \\
\hline \multicolumn{4}{|l|}{ Primary tumor } \\
\hline $\mathrm{pT} 2$ & $1(14 \%)$ & $4(22 \%)$ & \multirow[t]{2}{*}{0.9999} \\
\hline pT3 & $6(86 \%)$ & $14(78 \%)$ & \\
\hline \multicolumn{4}{|c|}{ Regional lymph nodes } \\
\hline $\mathrm{pN} 0$ & $1(14 \%)$ & $5(28 \%)$ & \multirow[t]{3}{*}{0.1316} \\
\hline $\mathrm{pN} 1$ & $5(71 \%)$ & $5(28 \%)$ & \\
\hline $\mathrm{pN} 2$ & $1(14 \%)$ & $8(44 \%)$ & \\
\hline \multicolumn{4}{|l|}{ pTNM stage } \\
\hline I & $1(14 \%)$ & $2(11 \%)$ & \multirow[t]{3}{*}{0.3962} \\
\hline II & $0(0 \%)$ & $4(22 \%)$ & \\
\hline III & $6(86 \%)$ & $12(67 \%)$ & \\
\hline \multicolumn{4}{|c|}{ Lymphatic invasion } \\
\hline No & $1(14 \%)$ & $4(22 \%)$ & \multirow[t]{2}{*}{0.9999} \\
\hline Yes & $6(86 \%)$ & $14(78 \%)$ & \\
\hline \multicolumn{4}{|l|}{ Venous invsion } \\
\hline No & $1(14 \%)$ & $1(6 \%)$ & \multirow[t]{2}{*}{0.4900} \\
\hline Yes & $6(86 \%)$ & $17(94 \%)$ & \\
\hline
\end{tabular}

not only on a total mesorectal excision, but also on intrapelvic lateral LN dissection, whereas the latter procedure is performed only rarely in Western countries. There are several possible reasons for this difference of opinion regarding the clinical significance of an iliac lymphadenectomy. The clinical importance of the lateral spread of lower rectal cancer was first demonstrated in the 1950s (23). Since then, LPLD was performed for middle or lower rectal cancer in Japan, because the incidence of lateral $\mathrm{LN}$ metastasis in patients with lower rectal cancer is high $(13-18 \%)(7,24,25)$. This procedure is associated with a lower local recurrence rate than conventional surgery. However, due to the high incidence of complications with LPLD, including sexual and urinary dysfunction (8), and the poor prognosis of patients with lateral LN metastasis (5-year survival rate, $38-42 \%)(7,24,25)$, this operation has only been performed in Japan. As a result, it is necessary to restrict the indications of LPLD for advanced lower rectal cancer.

The $\mathrm{SN}$ is defined as the first node in the regional lymphatic basin that drains the primary tumor and either a radionucleotide tracer or vital dye has been used to detect it.
The clinical applicability of SNNS and radio-guided SN detection to gastrointestinal tract cancer has been extensively investigated (13-16). The usefulness of dye-guided SN detection in colon cancer has already been reported (26). These studies demonstrated the high efficacy (70-99\%) of $\mathrm{SN}$. However, we were able to identify SNs in only 7 of the 25 patients (success rate, 28\%). In comparison to these studies, our detection rate of SNs is very low. One of the reasons for this is the degree of TNM stage. We investigated the SNs in advanced rectal cancer and therefore the detection rate may be low. Another reason may be that the use of dye followed by intraoperative tracing was impracticable, because of the close vicinity of the pelvic region and the narrow space around the mesorectal tissue (27). A final reason, may be due to the method used to detect SNs, namely, we detected SNs macroscopically.

The presence of LN metastasis is usually assessed by hematoxylin and eosin staining. Micrometastasis is generally evaluated by IHC or RT-PCR. Several investigators have emphasized the significance of LN micrometastasis as diagnosed by RT-PCR in colorectal cancer (CRC) $(20,22)$. In 
the present study, we used RNA rather than DNA to investigate the existence of micrometastases. DNA-based methods have the limitation that mutations of K-ras and/or p53 are found in subsets of CRC, but not in all cases, whereas CEA is expressed exclusively by primary CRC tissues $(20,28)$. Another reason for using this technique is that tumor cells detected by RT-PCR are very likely to be viable since mRNA is extremely unstable. In contrast, there is concern that mutated DNA found in regional LNs might represent a fraction of free tumor DNA rather than being derived from viable cancer cells $(29,30)$. Although special techniques, which act as a supplement to light microscopy, such as IHC and RT-PCR analyses, may improve the accuracy of the pathological staging, these modalities tend to be rather expensive, labor intensive and time-consuming for each LN of the operative specimens. Consequently, the possibility of a focused analysis of one or a few LNs which can reliably predict the regional nodal status is certainly attractive (31).

In the present study, we designed an original CEA primer, since CEA is an epithelial-specific antigen that is expressed in most cancers and in normal gastrointestinal tissue (32). Many studies have detected LN micrometastasis in CRC using RT-PCR of CEA mRNA $(19,20,28)$. Our method was thus able to detect 100 cancer cells mixed with $10^{7}$ normal lymphocytes. However, this rate is not so high. This may be attributable to differences in the system of reverse transcription and the PCR conditions. The CEA transcripts used as a target for the amplification of PCR is expressed in both normal epithelial and CRC cells and, therefore, it is possible that the detection rate would increase with an increasing number of PCR cycles. It has been reported that the nested PCR technique, which can achieve a high sensitivity by the two-step amplification of CEA cDNA, results in a $100 \%$ detection rate in node-negative CRC patients (33).

Lateral LN metastasis is generally considered to be a sign of systemic disease due to its poor prognosis in affected patients. However, some patients survived $>5$ years without adjuvant therapy, thus suggesting that the spread to the lateral LNs may not always represent systemic disease and that some patients may be cured by extended surgery. Although extensive preoperative and intraoperative investigations for lateral LN spread were performed, its incidence is thought to be low. Therefore, we need to develop a method to accurately select the patients who should undergo LPLD. From the viewpoint of detecting LN metastasis, minimally invasive surgery with a personalized lymphadenectomy can be performed if SNNS is introduced to patients with advanced lower rectal cancer. We can detect the lateral SNs using ICG. The sensitivity of lateral LN metastasis was found to improve using a genetic diagnosis. However, the detection rate of SNs was very low, as a result we still have to develop a new method for detecting SNs.

The present study investigated the adequacy of the SN concept based on LN micrometastasis as determined by RT-PCR. No definitive conclusion was made as to whether micrometastasis by RT-PCR/IHC or macrometastasis by $\mathrm{HE}$ is a more effective diagnostic modality for SNs. However, if we judge SN metastasis by HE only, we may miss metastatic nonSNs. As a result, we consider RT-PCR to be better than HE in making a final decision regarding the SNs.

\section{References}

1. Chapuis PH, Dent OF, Fisher R, Newland RC, Pheils MT, Smyth E and Colquhoun K: A multivariate analysis of clinical and pathological variables in prognosis after resection of large bowel cancer. Br J Surg 72: 698-702, 1985.

2. Koyama Y and Kotake K: Overview of colorectal cancer in Japan: report from the registry of the Japanese society for cancer of the colon and rectum. Dis Colon Rectum 40 (Suppl 10): S2-S9, 1997.

3. Ratto C, Sofo L, Ippoliti M, Merico M, Doglietto GB and Crucitti F: Prognostic factors in colorectal cancer. Literature review for clinical application. Dis Colon Rectum 41: 1033-1049, 1998.

4. Gennari L, Doci R and Rossetti C: Prognostic factors in colorectal cancer. Hepatogastroenterology 47: 310-314, 2000.

5. Nelson H, Petrelli N, Carlin A, Couture J, Fleshman J, Guillem J, Miedema B, Ota D and Sargent D: National Cancer Institute Expert Panel: Guidelines 2000 for colon and rectal cancer surgery. J Natl Cancer Inst 93: 583-596, 2001.

6. Welch JP and Donaldson GA: The clinical correlation of an autopsy study of recurrent colorectal cancer. Ann Surg 189: 496-502, 1979.

7. Moriya Y, Hojo K, Sawada T and Koyama Y: Significance of lateral node dissection for advanced rectal carcinoma at or below the peritoneal reflection. Dis Colon Rectum 32: 307-315, 1989.

8. Hojo K, Sawada T and Moriya Y: An analysis of survival and voiding, sexual function after wide iliopelvic lymphadenectomy in patients with carcinoma of the rectum, compared with conventional lymphadenectomy. Dis Colon Rectum 32: 128-133, 1989.

9. Morita T, Murata A, Koyama M, Totsuka E and Sasaki M: Current status of autonomic nerve-preserving surgery for mid and lower rectal cancers: Japanese experience with lateral node dissection. Dis Colon Rectum 46 (Suppl 10): S78-S87, 2003.

10. Morton DL, Wen DR, Wong JH, Economou JS, Cagle LA, Storm FK, Foshag LJ and Cochran AJ: Technical details of intraoperative lymphatic mapping for early stage melanoma. Arch Surg 127: 392-399, 1992

11. Veronesi U, Paganelli G, Galimberti V, Viale G, Zurrida S, Bedoni M, Costa A, de Cicco C, Geraghty JG, Luini A, Sacchini V and Veronesi P: Sentinel-node biopsy to avoid axillary dissection in breast cancer with clinically negative lymph-nodes. Lancet 349: 1864-1867, 1997.

12. Edwards MJ, Martin KD and McMasters KM: Lymphatic mapping and sentinel lymph node biopsy in the staging of melanoma. Surg Oncol 7: 51-57, 1998.

13. Kitagawa Y, Fujii H, Mukai M, Kubo A and Kitajima M: Current status and future prospects of sentinel node navigational surgery for gastrointestinal cancers. Ann Surg Oncol 11 (Suppl 3): 242S-244S, 2004.

14. Hiratsuka M, Miyashiro I, Ishikawa O, Furukawa H, Motomura K, Ohigashi H, Kameyama M, Sasaki Y, Kabuto T, Ishiguro S, Imaoka $S$ and Koyama $H$ : Application of sentinel node biopsy to gastric cancer surgery. Surgery 129: 335-340, 2001.

15. Saha S, Wiese D, Badin J, Beutler T, Nora D, Ganatra BK, Desai D, Kaushal S, Nagaraju M, Arora M and Singh T: Technical details of sentinel lymph node mapping in colorectal cancer and its impact on staging. Ann Surg Oncol 7: 120-124, 2000.

16. Joosten JJ, Strobbe LJ, Wauters CA, Pruszczynski M, Wobbes T and Ruers TJ: Intraoperative lymphatic mapping and the sentinel node concept in colorectal carcinoma. Br J Surg 86: 482-486, 1999.

17. Greenson JK, Isenhart CE, Rice R, Mojzisik C, Houchens D and Martin EW Jr: Identification of occult micrometastases in pericolic lymph nodes of Duke's B colorectal cancer patients using monoclonal antibodies against cytokeratin and CC49. Correlation with long-term survival. Cancer 73: 563-569, 1994.

18. Isaka N, Nozue M, Doy M and Fukao K: Prognostic significance of perirectal lymph node micrometastases in Dukes' B rectal carcinoma: an immunohistochemical study by CAM5.2. Clin Cancer Res 5: 2065-2068, 1999.

19. Liefers GJ, Cleton-Jansen AM, van de Velde CJ, Hermans J, van Krieken JH, Cornelisse CJ and Tollenaar RA: Micrometastases and survival in stage II colorectal cancer. N Engl J Med 339: 223-228, 1998. 
20. Noura S, Yamamoto H, Ohnishi T, Masuda N, Matsumoto T, Takayama O, Fukunaga H, Miyake Y, Ikenaga M, Ikeda M, Sekimoto M, Matsuura N and Monden M: Comparative detection of lymph node micrometastases of stage II colorectal cancer by reverse transcriptase polymerase chain reaction and immunohistochemistry. J Clin Oncol 20: 4232-4241, 2002.

21. Sobin LH and Witedkind CH: UICC TNM classification of malignant tumors. 5th edition, Wiley-Liss, New York, pp66-69, 1997.

22. Pilipshen SJ, Heilweil M, Quan SH, Sternberg SS and Enker WE: Patterns of pelvic recurrence following definitive resections of rectal cancer. Cancer 53: 1354-1362, 1984.

23. Sauer I and Bacon HE: Influence of lateral spread of cancer of the rectum on radicability of operation and prognosis. Am J Surg 81: $111-120,1951$.

24. Mori T, Takahashi K and Yasuno M: Radical resection with autonomic nerve preservation and lymph node dissection techniques in lower rectal cancer surgery and its results: the impact of lateral lymph node dissection. Langenbecks Arch Surg 383: 409-415, 1998.

25. Ueno H, Mochizuki H, Hashiguchi Y, Ishiguro M, Miyoshi M, Kajiwara Y, Sato T, Shimazaki $\mathrm{H}$ and Hase K: Potential prognostic benefit of lateral pelvic node dissection for rectal cancer located below the peritoneal reflection. Ann Surg 245: 80-87, 2007.

26. Saha S, Nora D, Wong JH and Weise D: Sentinel lymph node mapping in colorectal cancer - a review. Surg Clin North Am 80: 1811-1819, 2000

27. Bembenek A, Rau B, Moesta T, Markwardt J, Ulmer C, Gretschel S, Schneider U, Slisow W and Schlag PM: Sentinel lymph node biopsy in rectal cancer - not yet ready for routine clinical use. Surgery 135: 498-505, 2004.
28. Miyake Y, Yamamoto H, Fujiwara Y, Ohue M, Sugita Y, Tomita N, Sekimoto M, Matsuura N, Shiozaki H and Monden M: Extensive micrometastases to lymph nodes as a marker for rapid recurrence of colorectal cancer: a study of lymphatic mapping. Clin Cancer Res 7: 1350-1357, 2001.

29. Fujiwara Y, Chi DD, Wang H, Keleman P, Morton DL, Turner R and Hoon DS: Plasma DNA microsatellites as tumor-specific markers and indicators of tumor progression in melanoma patients. Cancer Res 59: 1567-1571, 1999.

30. Chen X, Bonnefoi H, Diebold-Berger S, Lyautey J, Lederrey C, Faltin-Traub E, Stroun M and Anker P: Detecting tumor-related alterations in plasma or serum DNA of patients diagnosed with breast cancer. Clin Cancer Res 5: 2297-2303, 1999.

31. Bertoglio S, Sandrucci S, Percivale P, Goss M, Gipponi M, Moresco L, Mussa B and Mussa A: Prognostic value of sentinel lymph node biopsy in the pathologic staging of colorectal cancer patients. J Surg Oncol 85: 166-170, 2004.

32. Gerhard M, Juhl H, Kalthoff H, Schreiber HW, Wagener C and Neumaier M: Specific detection of carcinoembryonic antigenexpressing tumor cells in bone marrow aspirates by polymerase chain reaction. J Clin Oncol 12: 725-729, 1994.

33. Futamura M, Takagi Y, Koumura H, Kida H, Tanemura H, Shimokawa K and Saji S: Spread of colorectal cancer micrometastases in regional lymph nodes by reverse transcriptasepolymerase chain reactions for carcinoembryonic antigen and cytokeratin 20. J Surg Oncol 68: 34-40, 1998. 